\title{
NOMURA ALGEBRAS OF NONSYMMETRIC HADAMARD MODELS
}

\author{
TAKUYA IKUTA AND AKIHIRO MUNEMASA
}

\begin{abstract}
We show that the Nomura algebra of the nonsymmetric Hadamard model coincides with the Bose-Mesner algebra of the directed Hadamard graph.
\end{abstract}

\section{INTRODUCTION}

Spin models for link invariants were introduced by Jones [7], and their connection to combinatorics was revealed first in [4]. Jaeger and Nomura [6] constructed nonsymmetric spin models for link invariants from Hadamard matrices, and showed that these models give link invariants which depend nontrivially on link orientation. These models are a modification of the Hadamard model originally constructed by Nomura [9. Jaeger and Nomura also pointed out a similarity between the association scheme of a Hadamard graph and the association scheme containing their new nonsymmetric spin model.

Nomura [10], and later Jaeger, Matsumoto and Nomura [5] introduced an algebra called the Nomura algebra of a type II matrix $W$, and showed that this algebra coincides with the Bose-Mesner algebra of some self-dual association schemes when $W$ is a spin model. By [5] the Nomura algebra of the Hadamard model coincides with the BoseMesner algebra of the corresponding Hadamard graph.

The purpose of this paper is to determine the Nomura algebra of a nonsymmetric Hadamard model to be the Bose-Mesner algebra of the corresponding directed Hadamard graph. We also show that the directed Hadamard graph can be constructed from the ordinary Hadamard graphs by means of a general method given by Klin, Muzychuk, Pech, Woldar and Zieschang [8].

\section{Preliminaries on Nomura algebras}

Let $X$ be a finite set with $k$ elements. We denote by $\operatorname{Mat}_{X}(\mathbf{C})$ the algebra of square matrices with complex entries whose rows and columns are indexed by $X$. We also denote by $\operatorname{Mat}_{X}\left(\mathbf{C}^{*}\right)$ the subset

Date: October 25, 2011. 
of $\operatorname{Mat}_{X}(\mathbf{C})$ consisting of matrices all of whose entries are nonzero. For $W \in \operatorname{Mat}_{X}(\mathbf{C})$ and $x, y \in X$, the $(x, y)$-entry of $W$ is denoted by $W(x, y)$.

A type II matrix is a matrix $W \in \operatorname{Mat}_{X}\left(\mathbf{C}^{*}\right)$ which satisfies the type II condition:

$$
\sum_{x \in X} \frac{W(a, x)}{W(b, x)}=k \delta_{a, b} \quad(\text { for all } a, b \in X) .
$$

For a type II matrix $W \in \operatorname{Mat}_{X}\left(\mathbf{C}^{*}\right)$ and $a, b \in X$, we define a column vector $Y_{a b} \in \mathbf{C}^{X}$ whose $x$-entry is given by

$$
Y_{a b}(x)=\frac{W(x, a)}{W(x, b)} .
$$

The Nomura algebra $\mathcal{N}(W)$ of $W$ is defined by

$\mathcal{N}(W)=\left\{M \in \operatorname{Mat}_{n}\left(\mathbf{C}^{*}\right) \mid Y_{a b}\right.$ is an eigenvector for $M$ for all $\left.a, b \in X\right\}$.

A type II matrix $W$ is called a spin model if it satisfies the type III condition:

$$
\left.\sum_{x \in X} \frac{W(a, x) W(b, x)}{W(c, x)}=d \frac{W(a, b)}{W(a, c) W(c, b)} \quad \text { (for all } a, b, c \in X\right),
$$

where $d^{2}=k$. It is shown in [5. Theorem 11] that if $W$ is a spin model, then $\mathcal{N}(W)$ is the Bose-Mesner algebra of some self-dual association scheme.

We shall associate with $W$ an undirected graph $G$ on the vertex set $X \times X$. Given two column vectors $T, T^{\prime}$ indexed by $X$, we write $\left\langle T, T^{\prime}\right\rangle$ for their Hermitian scalar product $\sum_{x \in X} T(x) \overline{T^{\prime}(x)}$. Two distinct vertices $(a, b),(c, d)$ will be adjacent in $G$ iff $\left\langle Y_{a b}, Y_{c d}\right\rangle \neq 0$. For a subset $C$ of $X \times X$, we denote by $A(C)$ the matrix in $\operatorname{Mat}_{X}(\mathbf{C})$ with $(a, b)$-entry equal to 1 if $(a, b) \in C$ and to 0 otherwise. Then we have the following:

Theorem 1. [5, Theorem 5(iii)] Let $C_{1}, \ldots, C_{p}$ be the connected components of $G$. Then the algebra $\mathcal{N}\left(W^{T}\right)$ has a basis $\left\{A\left(C_{i}\right) \mid i=1, \ldots, p\right\}$.

\section{Hadamard graphs and directed Hadamard graphS}

In this section, we define the adjacency matrices of Hadamard graphs and directed Hadamard graphs, and give the association schemes determined by them. We refer the reader to [1, Theorem 1.8.1] for properties of Hadamard graphs, and to [2] for background materials in the theory of association schemes. 
Let $k$ be a positive integer, and let $H \in \operatorname{Mat}_{X}(\mathbf{C})$ be a Hadamard matrix of order $k$. We denote by $I_{n}$ the identity matrix of order $n$, and we omit $n$ if $n=k$. Let $J \in \operatorname{Mat}_{X}(\mathbf{C})$ be the all 1's matrix. We define

$$
\begin{aligned}
A_{0}= & I_{4 k}, \\
A_{1}= & {\left[\begin{array}{cccc}
0 & 0 & \frac{1}{2}(J+H) & \frac{1}{2}(J-H) \\
0 & 0 & \frac{1}{2}(J-H) & \frac{1}{2}(J+H) \\
\frac{1}{2}\left(J+H^{T}\right) & \frac{1}{2}\left(J-H^{T}\right) & 0 & 0 \\
\frac{1}{2}\left(J-H^{T}\right) & \frac{1}{2}\left(J+H^{T}\right) & 0 & 0
\end{array}\right], } \\
A_{2}= & {\left[\begin{array}{cccc}
J-I & J-I & 0 & 0 \\
J-I & J-I & 0 & 0 \\
0 & 0 & J-I & J-I \\
0 & 0 & J-I & J-I
\end{array}\right], } \\
A_{3}= & {\left[\begin{array}{ccccc}
0 & 0 & \frac{1}{2}(J-H) & \frac{1}{2}(J+H) \\
\frac{1}{2}\left(J-H^{T}\right) & \frac{1}{2}\left(J+H^{T}\right) & \frac{1}{2}(J+H) & \frac{1}{2}(J-H) \\
\frac{1}{2}\left(J+H^{T}\right) & \frac{1}{2}\left(J-H^{T}\right) & 0
\end{array}\right], } \\
A_{4}= & {\left[\begin{array}{llll}
0 & I & 0 & 0 \\
I & 0 & 0 & 0 \\
0 & 0 & 0 & I \\
0 & 0 & I & 0
\end{array}\right] . }
\end{aligned}
$$

The matrices $\left\{A_{i}\right\}_{i=0}^{4}$ are the distance matrices of the Hadamard graph associated to the Hadamard matrix $H$. Since the Hadamard graph is distance-regular (see [1, Theorem 1.8.1]),

$$
\mathcal{A}=\operatorname{span}\left\{A_{0}, A_{1}, A_{2}, A_{3}, A_{4}\right\}
$$

is closed under the matrix multiplication. This algebra is called the Bose-Mesner algebra of the Hadamard graph.

The directed Hadamard graph associated with a Hadamard matrix $H$ is the digraph with adjacency matrix

$$
A_{1}^{\prime}=\left[\begin{array}{cccc}
0 & 0 & \frac{1}{2}(J+H) & \frac{1}{2}(J-H) \\
0 & 0 & \frac{1}{2}(J-H) & \frac{1}{2}(J+H) \\
\frac{1}{2}\left(J-H^{T}\right) & \frac{1}{2}\left(J+H^{T}\right) & 0 & 0 \\
\frac{1}{2}\left(J+H^{T}\right) & \frac{1}{2}\left(J-H^{T}\right) & 0 & 0
\end{array}\right] .
$$

The matrix $A_{1}^{\prime}$ generates a Bose-Mesner algebra $\mathcal{A}^{\prime}$ with basis $A_{0}, A_{1}^{\prime}, A_{2}, A_{3}^{\prime}=$ $A_{1}^{\prime T}, A_{4}$ (see [6]). We shall index the rows and columns of the matrices $A_{1}$ and $A_{1}^{\prime}$ by $X \times(\mathbf{Z} / 2 \mathbf{Z})^{2}$, in the order $X \times\{(0,0)\}, X \times\{(0,1)\}$, $X \times\{(1,0)\}, X \times\{(1,1)\}$. Then $\mathcal{A}$ is the Bose-Mesner algebra of the 
association scheme on $X \times(\mathbf{Z} / 2 \mathbf{Z})^{2}$ with relations

$$
\begin{aligned}
R_{0}= & \left\{((a, \alpha),(a, \alpha)) \mid(a, \alpha) \in X \times(\mathbf{Z} / 2 \mathbf{Z})^{2}\right\}, \\
R_{1}= & \left\{((a, \alpha),(b, \beta)) \mid\left(\alpha_{1}, \beta_{1}\right)=(0,1), H(a, b)=(-1)^{\alpha_{2}+\beta_{2}}\right\} \\
& \cup\left\{((a, \alpha),(b, \beta)) \mid\left(\alpha_{1}, \beta_{1}\right)=(1,0), H(b, a)=(-1)^{\alpha_{2}+\beta_{2}}\right\}, \\
R_{2}= & \left\{((a, \alpha),(b, \beta)) \mid \alpha_{1}=\beta_{1}, a \neq b\right\}, \\
R_{3}= & \left\{((a, \alpha),(b, \beta)) \mid\left(\alpha_{1}, \beta_{1}\right)=(0,1), H(a, b)=(-1)^{\alpha_{2}+\beta_{2}+1}\right\} \\
& \cup\left\{((a, \alpha),(b, \beta)) \mid\left(\alpha_{1}, \beta_{1}\right)=(1,0), H(b, a)=(-1)^{\alpha_{2}+\beta_{2}+1}\right\}, \\
R_{4}= & \left\{((a, \alpha),(a, \beta)) \mid \alpha_{1}=\beta_{1}, \alpha_{2} \neq \beta_{2}\right\} .
\end{aligned}
$$

Also, $\mathcal{A}^{\prime}$ is the Bose-Mesner algebra of an association scheme on $X \times$ $(\mathbf{Z} / 2 \mathbf{Z})^{2}$ with relations $R_{0}, R_{1}^{\prime}, R_{2}, R_{3}^{\prime}, R_{4}$, where

$$
\begin{aligned}
R_{1}^{\prime}= & \left\{((a, \alpha),(b, \beta)) \mid\left(\alpha_{1}, \beta_{1}\right)=(0,1), H(a, b)=(-1)^{\alpha_{2}+\beta_{2}}\right\} \\
& \cup\left\{((a, \alpha),(b, \beta)) \mid\left(\alpha_{1}, \beta_{1}\right)=(1,0), H(b, a)=(-1)^{\alpha_{2}+\beta_{2}+1}\right\}, \\
R_{3}^{\prime}= & \left\{((a, \alpha),(b, \beta)) \mid\left(\alpha_{1}, \beta_{1}\right)=(0,1), H(a, b)=(-1)^{\alpha_{2}+\beta_{2}+1}\right\} \\
& \cup\left\{((a, \alpha),(b, \beta)) \mid\left(\alpha_{1}, \beta_{1}\right)=(1,0), H(b, a)=(-1)^{\alpha_{2}+\beta_{2}}\right\} .
\end{aligned}
$$

Let

$$
\begin{aligned}
Z_{0} & =X \times\{0\} \times \mathbf{Z} / 2 \mathbf{Z}, \\
Z_{1} & =X \times\{1\} \times \mathbf{Z} / 2 \mathbf{Z}, \\
Z & =Z_{0} \cup Z_{1} \\
R_{i}^{0} & =R_{i} \cap\left(Z_{0} \times Z\right), \\
R_{i}^{1} & =R_{i} \cap\left(Z_{1} \times Z\right) .
\end{aligned}
$$

Let

$$
\mathcal{R}=\left\{R_{i}^{0} \mid i=0, \ldots, 4\right\} \cup\left\{R_{i}^{1} \mid i=0, \ldots, 4\right\} .
$$

Then $\mathcal{R}$ is a coherent configuration in the sense of [3]. Let $R_{1}^{\prime}=R_{1}^{0} \cup R_{3}^{1}$, $R_{3}^{\prime}=R_{1}^{1} \cup R_{3}^{0}$. Then

$$
R_{i}^{\lambda} R_{j}^{\mu}=\delta_{i+\lambda \bmod 2, \mu} \sum_{k \equiv i+j} p_{(\bmod 2)}^{k} R_{k}^{\lambda} .
$$

It follows that the permutation $\rho$ of $\mathcal{R}$ defined by

$$
\rho\left(R_{i}^{\delta}\right)= \begin{cases}R_{3}^{1-\delta} & \text { if } i=1, \\ R_{1}^{1-\delta} & \text { if } i=3, \\ R_{i}^{1-\delta} & \text { otherwise }\end{cases}
$$

is an algebraic automorphism of the coherent configuration $\mathcal{R}$ in the sense of [8]. Since the relations $\mathcal{R}^{\prime}=\left\{R_{0}, R_{1}^{\prime}, R_{2}, R_{3}^{\prime}, R_{4}\right\}$ are obtained 
by fusing $\rho$-orbits, the fact that $\mathcal{R}^{\prime}$ forms an association scheme follows also from the general theory given in [8, Subsection 2.6].

\section{Symmetric And nOnSymmetric Hadamard MODELS}

Throughout this section, we assume that $k$ is an integer with $k \geq 4$. Let $u$ be a complex number satisfying

$$
k=\left(u^{2}+u^{-2}\right)^{2}
$$

A Potts model $A \in \operatorname{Mat}_{X}\left(\mathbf{C}^{*}\right)$ is defined as

$$
A=u^{3} I-u^{-1}(J-I) .
$$

Let $H \in \operatorname{Mat}_{X}(\mathbf{C})$ be a Hadamard matrix of order $k$. In [9], 6], the following two spin models are given:

$$
\begin{aligned}
W & =\left[\begin{array}{cccc}
A & A & \omega H & -\omega H \\
A & A & -\omega H & \omega H \\
\omega H^{T} & -\omega H^{T} & A & A \\
-\omega H^{T} & \omega H^{T} & A & A
\end{array}\right] \\
& =u^{3} A_{0}+\omega A_{1}-u^{-1} A_{2}-\omega A_{3}+u^{3} A_{4}, \\
W^{\prime} & =\left[\begin{array}{cccc}
A & A & \xi H & -\xi H \\
A & A & -\xi H & \xi H \\
-\xi H^{T} & \xi H^{T} & A & A \\
\xi H^{T} & -\xi H^{T} & A & A
\end{array}\right] \\
& =u^{3} A_{0}+\xi A_{1}^{\prime}-u^{-1} A_{2}-\xi A_{3}^{\prime}+u^{3} A_{4},
\end{aligned}
$$

where $\omega$ is a 4 -th root of unity, $\xi$ is a primitive 8-th root of unity, respectively. We index the rows and columns of the matrices (5), (6) by $X \times(\mathbf{Z} / 2 \mathbf{Z})^{2}$ as in Section 3. The spin models (5) and (6) are called a Hadamard model and a nonsymmetric Hadamard model, respectively. From [5, Subsection 5.5] we have

$$
\mathcal{N}(W)=\mathcal{A}
$$


In order to determine the Nomura algebra $\mathcal{N}\left(W^{\prime}\right)$, we consider the normalized version of the matrices (15), (6) :

$$
\begin{aligned}
\tilde{W} & =\left[\begin{array}{cccc}
A & A & H & -H \\
A & A & -H & H \\
H^{T} & -H^{T} & A & A \\
-H^{T} & H^{T} & A & A
\end{array}\right], \\
\tilde{W}^{\prime} & =\left[\begin{array}{cccc}
A & A & H & -H \\
A & A & -H & H \\
i H^{T} & -i H^{T} & A & A \\
-i H^{T} & i H^{T} & A & A
\end{array}\right],
\end{aligned}
$$

where $i=-\xi^{2}$ is a primitive 4 th root of unity. Then $\mathcal{N}(W)=\mathcal{N}(\tilde{W})$ and $\mathcal{N}\left(W^{\prime}\right)=\mathcal{N}\left(\tilde{W}^{\prime}\right)$, since

$$
\tilde{W}=\left[\begin{array}{cccc}
I & & & 0 \\
& I & & \\
& & \omega I & \\
0 & & & \omega I
\end{array}\right] W\left[\begin{array}{cccc}
I & & & 0 \\
& I & & \\
& & \omega^{-1} I & \\
0 & & & \omega^{-1} I
\end{array}\right]
$$

if $\omega^{2}=1$,

$$
\tilde{W}=\left[\begin{array}{cccc}
I & & & 0 \\
& I & & \\
& & \omega I & \\
0 & & & \omega I
\end{array}\right] W\left[\begin{array}{ccc}
I & & 0 \\
I & & \\
& \omega^{-1} I & \\
0 & & \omega^{-1} I
\end{array}\right]
$$

if $\omega^{2}=-1$, and

$$
\tilde{W}^{\prime}=\left[\begin{array}{llll}
I & & & 0 \\
& I & & \\
& & \xi I & \\
0 & & & \xi I
\end{array}\right] W^{\prime}\left[\begin{array}{cccc}
I & & & 0 \\
& I & & \\
& & \xi^{-1} I & \\
0 & & & \xi^{-1} I
\end{array}\right]
$$

(see [5, Propositions 2 and 3]).

Define column vectors $Y_{a b}^{\alpha \beta}, Y_{a b}^{\alpha \beta}$ whose $x$-entries are given by

$$
\begin{aligned}
Y_{a b}^{\alpha \beta}(x) & =\frac{\tilde{W}(x,(a, \alpha))}{\tilde{W}(x,(b, \beta))}, \\
Y_{a b}^{\prime \alpha \beta}(x) & =\frac{\tilde{W}^{\prime}(x,(a, \alpha))}{\tilde{W}^{\prime}(x,(b, \beta))}
\end{aligned}
$$

for $(a, \alpha),(b, \beta) \in X \times(\mathbf{Z} / 2 \mathbf{Z})^{2}, x \in X \times(\mathbf{Z} / 2 \mathbf{Z})^{2}$. 
Lemma 2. Let $G$ and $G^{\prime}$ be the graphs with the same vertex set $(X \times$ $\left.(\mathbf{Z} / 2 \mathbf{Z})^{2}\right)^{2}$, where two distinct vertices $((a, \alpha),(b, \beta)),\left(\left(a^{\prime}, \alpha^{\prime}\right),\left(b^{\prime}, \beta^{\prime}\right)\right)$ are adjacent whenever

$$
\begin{gathered}
\left\langle Y_{a b}^{\alpha \beta}, Y_{a^{\prime} b^{\prime}}^{\alpha^{\prime} \beta^{\prime}}\right\rangle \neq 0, \\
\left\langle Y_{a b}^{\alpha \beta}, Y_{a^{\prime} b^{\prime}}^{\alpha^{\prime} \beta^{\prime}}\right\rangle \neq 0,
\end{gathered}
$$

respectively. Let $K_{j}(j=1, \ldots, p)$ (resp. $\left.K_{j}^{\prime}\left(j=1, \ldots, p^{\prime}\right)\right)$ be the connected components of $G$ (resp. $\left.G^{\prime}\right)$. Then $\mathcal{N}(W)$ (resp. $\left.\mathcal{N}\left(W^{\prime}\right)\right)$ is spanned by $\left\{A\left(K_{j}\right) \mid j=1, \ldots, p\right\}$ (resp. $\left.\left\{A\left(K_{j}^{\prime}\right) \mid j=1, \ldots, p^{\prime}\right\}\right)$.

Proof. By [5, Section 5.5], (77) holds regardless of the value of $\omega$, so we have $\mathcal{N}(W)=\mathcal{N}(\tilde{W})$. Since $\tilde{W}=\tilde{W}^{T}$, the result for $\mathcal{N}(W)$ follows immediately from Theorem 1 .

Since

$$
\tilde{W}^{\prime T}=\left[\begin{array}{cc}
I_{2 k} & 0 \\
0 & -\xi^{-1} I_{2 k}
\end{array}\right] W^{\prime}\left[\begin{array}{cc}
I_{2 k} & 0 \\
0 & -\xi I_{2 k}
\end{array}\right],
$$

we have $\mathcal{N}\left(W^{\prime}\right)=\mathcal{N}\left(\tilde{W}^{\prime T}\right)$ by [5, Proposition 2]. Thus, the result for $\mathcal{N}\left(W^{\prime}\right)$ follows also from Theorem 1 .

Let

$$
D=\left[\begin{array}{llll}
I & & & 0 \\
& I & & \\
& & i I & \\
0 & & & i I
\end{array}\right] \in \operatorname{Mat}_{X \times(\mathbf{Z} / 2 \mathbf{Z})^{2}}(\mathbf{C}) .
$$

Lemma 3. For $(a, \alpha),(b, \beta) \in X \times(\mathbf{Z} / 2 \mathbf{Z})^{2}$,

$$
Y_{a b}^{\alpha \beta}= \begin{cases}Y_{a b}^{\alpha \beta} & \text { if } \alpha_{1}=\beta_{1}, \\ D Y_{a b}^{\alpha \beta} & \text { if }\left(\alpha_{1}, \beta_{1}\right)=(0,1), \\ D^{-1} Y_{a b}^{\alpha \beta} & \text { if }\left(\alpha_{1}, \beta_{1}\right)=(1,0) .\end{cases}
$$

Proof. Immediate from the definitions (8), (91), (10) and (11).

Lemma 4. Let $\tau$ denote the permutation of $(\mathbf{Z} / 2 \mathbf{Z})^{2}$ defined by

$$
\tau\left(\alpha_{1}, \alpha_{2}\right)=\left(\alpha_{1}, \alpha_{1}+\alpha_{2}\right)
$$

and let $\sigma$ denote the permutation of $\left(X \times(\mathbf{Z} / 2 \mathbf{Z})^{2}\right)^{2}$ defined by

$$
\sigma((a, \alpha),(b, \beta))=((a, \tau(\alpha)),(b, \beta)) .
$$

Then $\sigma\left(R_{1}\right)=R_{1}^{\prime}$ and $\sigma\left(R_{3}\right)=R_{3}^{\prime}$.

Proof. Immediate from the definitions of $R_{1}, R_{3}, R_{1}^{\prime}$ and $R_{3}^{\prime}$.

Lemma 5. For $(a, \alpha),(b, \beta) \in X \times(\mathbf{Z} / 2 \mathbf{Z})^{2}$,

$$
Y_{a b}^{\tau(\alpha) \beta}=\left(-D^{2}\right)^{\alpha_{1}} Y_{a b}^{\alpha \beta} .
$$


Proof. Since $\tau(\alpha)=\alpha$ when $\alpha_{1}=0$, the result holds in this case. If $\alpha_{1}=1$, then $\tau(\alpha)=\left(1,1+\alpha_{2}\right)$. Since the $(a, \alpha)$-column and $(a, \tau(\alpha))$ column of $\tilde{W}$ differ by the left multiplication by $-D^{2}$, the results holds in this case as well.

Lemma 6. If $((a, \alpha),(b, \beta)),\left(\left(a^{\prime}, \alpha^{\prime}\right),\left(b^{\prime}, \beta^{\prime}\right)\right) \in R_{1} \cup R_{3}$, then

$$
\left\langle Y_{a b}^{\prime \tau(\alpha) \beta}, Y_{a^{\prime} b^{\prime}}^{\prime \tau\left(\alpha^{\prime}\right) \beta^{\prime}}\right\rangle=(-1)^{\alpha_{1}+\alpha_{1}^{\prime}}\left\langle Y_{a b}^{\alpha \beta}, Y_{a^{\prime} b^{\prime}}^{\alpha^{\prime} \beta^{\prime}}\right\rangle
$$

Proof. Using Lemmas 3 and 5, we have

$$
\begin{aligned}
& \left\langle Y_{a b}^{\prime \tau(\alpha) \beta}, Y_{a^{\prime} b^{\prime}}^{\tau\left(\alpha^{\prime}\right) \beta^{\prime}}\right\rangle \\
& =\left\{\begin{array}{lll}
\left\langle D Y_{a b}^{\tau(\alpha) \beta}, D Y_{a^{\prime} b^{\prime}}^{\tau\left(\alpha^{\prime}\right) \beta^{\prime}}\right\rangle & \text { if }\left(\tau(\alpha)_{1}, \beta_{1}\right)=\left(\tau\left(\alpha^{\prime}\right)_{1}, \beta_{1}^{\prime}\right)=(0,1), \\
\left\langle D^{-1} Y_{a b}^{\tau(\alpha) \beta}, D^{-1} Y_{\left.a^{\prime} b^{\prime}\right) \beta^{\prime}}^{\tau\left(\alpha^{\prime}\right.}\right\rangle & \text { if }\left(\tau(\alpha)_{1}, \beta_{1}\right)=\left(\tau\left(\alpha^{\prime}\right)_{1}, \beta_{1}^{\prime}\right)=(1,0), \\
\left\langle D Y_{a b}^{\tau(\alpha) \beta}, D^{-1} Y_{\left.a^{\prime} \alpha^{\prime}\right)}^{\tau\left(\alpha^{\prime}\right.}\right\rangle & \text { if }\left(\tau(\alpha)_{1}, \beta_{1}\right)=\left(\beta_{1}^{\prime}, \tau\left(\alpha^{\prime}\right)_{1}\right)=(0,1), \\
\left\langle D^{-1} Y_{a b}^{\tau(\alpha) \beta}, D Y_{a^{\prime} b^{\prime}}^{\tau\left(\alpha^{\prime}\right) \beta^{\prime}}\right\rangle & \text { if }\left(\tau(\alpha)_{1}, \beta_{1}\right)=\left(\beta_{1}^{\prime}, \tau\left(\alpha^{\prime}\right)_{1}\right)=(1,0)
\end{array}\right. \\
& = \begin{cases}\left\langle Y_{a b}^{\tau(\alpha) \beta}, Y_{\left.a^{\prime} b^{\prime}\right) \beta^{\prime}}^{\tau\left(\alpha^{\prime}\right.}\right\rangle & \text { if }\left(\alpha_{1}, \beta_{1}\right)=\left(\alpha_{1}^{\prime}, \beta_{1}^{\prime}\right), \\
\left\langle D^{2} Y_{a b}^{\tau(\alpha) \beta}, Y_{a^{\prime} b^{\prime}}^{\tau\left(\alpha^{\prime}\right) \beta^{\prime}}\right\rangle & \text { otherwise }\end{cases} \\
& = \begin{cases}\left\langle\left(-D^{2}\right)^{\alpha_{1}} Y_{a b}^{\alpha \beta},\left(-D^{2}\right)^{\alpha_{1}^{\prime}} Y_{a^{\prime} b^{\prime}}^{\alpha^{\prime} \beta^{\prime}}\right\rangle & \text { if } \alpha_{1}=\alpha_{1}^{\prime}, \\
\left\langle D^{2}\left(-D^{2}\right)^{\alpha_{1}} Y_{a b}^{\alpha \beta},\left(-D^{2}\right)^{\alpha_{1}^{\prime}} Y_{a^{\prime} b^{\prime}}^{\alpha^{\prime} \beta^{\prime}}\right\rangle & \text { otherwise }\end{cases} \\
& = \begin{cases}\left\langle Y_{a b}^{\alpha \beta}, Y_{a^{\prime} b^{\prime}}^{\alpha^{\prime}}\right\rangle & \text { if } \alpha_{1}=\alpha_{1}^{\prime}, \\
-\left\langle Y_{a b}^{\alpha \beta}, Y_{a^{\prime} b^{\prime}}^{\alpha^{\prime} \beta^{\prime}}\right\rangle & \text { otherwise }\end{cases} \\
& =(-1)^{\alpha_{1}+\alpha_{1}^{\prime}}\left\langle Y_{a b}^{\alpha \beta}, Y_{a^{\prime} b^{\prime}}^{\alpha^{\prime} \beta^{\prime}}\right\rangle \text {. }
\end{aligned}
$$

Theorem 7. The Nomura algebra $\mathcal{N}\left(W^{\prime}\right)$ of the spin model $W^{\prime}$ coincides with the Bose-Mesner algebra $\mathcal{A}^{\prime}$ of the directed Hadamard graph.

Proof. Since $u^{4}=1$ or $|u|>1$, the coefficients $\left\{\xi,-u^{-1},-\xi, u^{3}\right\}$ of $W^{\prime}$ in $A_{1}^{\prime}, A_{2}, A_{3}^{\prime}, A_{4}$ are pairwise distinct. Since $W^{\prime} \in \mathcal{N}\left(W^{\prime}\right)$ by [5, Proposition 9], we obtain $A_{1}^{\prime}, A_{2}, A_{3}^{\prime}, A_{4} \in \mathcal{N}\left(W^{\prime}\right)$. By Lemma 2, this implies that each of $R_{1}^{\prime}, R_{2}, R_{3}^{\prime}, R_{4}$ is a union of connected components of $G^{\prime}$.

Since $\mathcal{N}(W)=\mathcal{A}$, Lemma 2 implies that $R_{0}, R_{1}, \ldots, R_{4}$ are the connected components of $G$. Observe

$$
R_{0} \cup R_{2} \cup R_{4}=\left\{((a, \alpha),(b, \beta)) \in\left(X \times(\mathbf{Z} / 2 \mathbf{Z})^{2}\right)^{2} \mid \alpha_{1}=\beta_{1}\right\} .
$$

By Lemma 3, we have

$$
Y_{a b}^{\alpha \beta}=Y_{a b}^{\alpha \beta} \quad \text { for }((a, \alpha),(b, \beta)) \in R_{0} \cup R_{2} \cup R_{4} .
$$


This implies that two graphs $G$ and $G^{\prime}$ have the same set of edges on $R_{0} \cup R_{2} \cup R_{4}$. Thus $R_{0}, R_{2}$ and $R_{4}$ are connected.

By Lemmas 4 and 6, there is an isomorphism $\sigma$ from the subgraph of $G$ induced by $R_{1} \cup R_{3}$, to the subgraph of $G^{\prime}$ induced by $R_{1}^{\prime} \cup R_{3}^{\prime}$, satisfying $\sigma\left(R_{1}\right)=R_{1}^{\prime}$ and $\sigma\left(R_{3}\right)=R_{3}^{\prime}$. Since $R_{1}$ and $R_{3}$ are connected components of $G, R_{1}^{\prime}$ and $R_{3}^{\prime}$ are connected.

Therefore, we have shown that $R_{0}, R_{1}^{\prime}, R_{2}, R_{3}^{\prime}, R_{4}$ are the connected components of $G^{\prime}$. The result now follows from Lemma 2 .

Remark 8. The condition of Theorem [7 does not hold when $k=1,2$. A direct calculation shows that $\mathcal{N}(W)=\mathcal{N}\left(W^{\prime}\right)$ is the Bose-Mesner algebra of the group association scheme of $\mathbf{Z} / 4 \mathbf{Z}$ when $k=1$, and that $\mathcal{N}(W) \cong \mathcal{N}\left(W^{\prime}\right)$ is the Bose-Mesner algebra of the group association scheme of $\mathbf{Z} / 8 \mathbf{Z}$ when $k=2$.

Acknowledgements. The authors would like to thank Mitsugu Hirasaka for bringing the article [8] to the authors' attention, and an anonymous referee for careful reading of the manuscript.

\section{REFERENCES}

[1] A.E. Brouwer, A.M. Cohen and A. Neumaier, Distance-Regular Graphs, Springer-Verlag, Heidelberg, 1989.

[2] E. Bannai and T. Ito, Algebraic Combinatorics I, Benjamin/Cummings, Menlo Park, 1984.

[3] D.G. Higman, Coherent configurations, I. Rend. Sem. Mat. Univ. Padova 44 (1970), 1-25.

[4] F. Jaeger, Strongly regular graphs and spin models for the Kauffman polynomial, Geom. Dedicata 44 (1992), 23-52.

[5] F. Jaeger, M. Matsumoto, and K. Nomura, Bose-Mesner algebras related to type II matrices and spin models, J. Algebraic Combin. 8 (1998), 39-72.

[6] F. Jaeger and K. Nomura, Symmetric versus non-symmetric spin models for link invariants, J. Algebraic Combin. 10 (1999), 241-278.

[7] V.F.R. Jones, On knot invariants related to some statistical mechanical models, Pacific J. Math. 137 (1989), 311-336.

[8] M. Klin, M. Muzychuk, C. Pech, A. Woldar, and P.-H. Zieschang, Association schemes on 28 points as mergings of a half-homogeneous coherent configuration, European J. Combin. 28 (2007), 1994-2025.

[9] K. Nomura, Spin models constructed from Hadamard matrices, J. Combin. Theory Ser. A 68 (1994), 251-261.

[10] K. Nomura, An algebra associated with a spin model, J. Algebraic Combin. 6 (1997), 53-58. 
Kobe Gakuin University, Chuo-Ku, Kobe, 650-8586 Japan

E-mail address: ikuta@law.kobegakuin.ac.jp

Graduate School of Information Sciences, Tohoku University, Sendai 980-8579, JAPAN

E-mail address: munemasa@math.is.tohoku.ac.jp 